\title{
Designing an Adaptive Emergency Warning System for Heterogeneous Environments
}

\author{
Gareth Tyson, John Bigham and Eliane Bodanese \\ Queen Mary University of London \\ London, UK
}

\author{
Pradipta Biswas and Pat Langdon \\ University of Cambridge \\ Cambridge, UK
}

\author{
Nadeem Akhtar \\ CEWIT \\ Chennai, India \\ Vineet Mimrot, Pratyay Mukhopadhyay and Vinay J. \\ Ribeiro \\ IIT Delhi \\ Delhi, India
}

\begin{abstract}
In this paper a novel cloud-based Emergency Warning System (EWS) is described, using India as a primary case study. India is a country with nearly two dozen officially recognized languages and divergent communication technologies spread across a large geographical area. As of yet, this diversity has made the deployment of a single nationwide EWS near impossible. To address this deficiency, we have developed an EWS with adaptation as a central design tenet. This adaptation occurs on three levels: Dissemination adaptation addresses civilians' heterogeneous communication technologies; information adaptation morphs warnings to best reflect the capabilities of these communication technologies and users; while presentation adaptation is used to render information to the user in the most appropriate manner. We have developed a full cloudbased prototype, including the full EWS infrastructure and a civilian Android app.
\end{abstract}

Keywords-Early Warning Systems; information adaptation; dissimination adaptation; heterogeneous communication environments;

\section{INTRODUCTION}

Natural disasters are becoming ever more prominent in our world today, increasing by over $400 \%$ in the last 20 years [1]. In 2009 alone, 335 natural disasters were recorded, causing $\$ 41.3$ billion in economic damages and affecting approximately 120 million people. This trend is constantly expanding with over 375 million people predicted to be affected by climate-related disasters per year by 2015 [2]. In addition, major social incidents such as terrorism are becoming increasingly frequent. For example, in India and the UK, such events have impacted entire cities, as exemplified by the Uttarakhand Flooding of 2013, the Carlisle Flooding of 2005, and the Terrorist Bombings of London in 2005. In light of this, it seems likely that nations will have to make increasing efforts to manage the challenges brought about by such devastating and unpredictable events.

To address this growing propensity, many are working on technological solutions to help deal with such situations. One technology that has received widespread attention is that of

This work has been sponsored by the EPSRC (UK) and the DST (India) through the Indo-UK Advanced Technology Centre (IU-ATC) project grant.
Emergency Warning Systems (EWS). These offer mechanisms to send information from authorities to civilians during times of crisis. Information of interest includes evacuation details, medical advice and precautionary actions to take. Popular techniques for dissemination are TV, radio, websites and increasingly social media such as Twitter [3].

Unfortunately, however, disseminating such information during disasters is fraught with challenges of a sociological (e.g. language), inclusivity (e.g. accessibility), and technological (e.g. resilience) nature. These issues are exacerbated by the unpredictable nature of most disasters. Seismic activity, for instance, can create notable disruptions in network infrastructure [4]. Co-location of physical infrastructure (e.g. network cables, electricity cables, gas pipes) can also magnify this impact, creating issues across multiple mediums.

This paper details a resilient cloud-based EWS for deployment in highly heterogeneous environments (both technologically and sociologically). At its heart is the concept of adaptation. We argue that an EWS must adapt on a variety of levels to ensure that civilians receive reliable, understandable and actionable warnings in times of crisis. Importantly, instead of focusing on infrastructure as the key component of resilience, we take a user-centric approach, designing methods to ensure information can always reach civilians in a usable form. Consequently, our EWS abstracts away from underlying delivery systems and adapts around the usage of any available mechanism to deliver the warning. This involves techniques such as cloud-based replication and intelligent service placement, alongside the exploitation of multiple redundant delivery channels (e.g. 3G, 4G, SMS, Bluetooth, IP). We similarly argue that adaptation should extend to the warning itself - it should not exist as an immutable block of bytes but, instead, be morphed to best convey its essence. For instance, a warning's essence may be to inform a civilian to leave an area; based on the user, this might be best conveyed via text, video, image or, alternatively, audio. This even extends to how the message is presented, e.g. 
translating between languages or using differing color schemes to reflect color blindness.

We begin by exploring the background to the topic, focusing on the needs of India as a driving case study. After this, we provide an overview of our EWS. Finally, we explain how the system exploits adaptation to address the various types of heterogeneity observed, specifically relating to (i) variations in runtime communications capabilities; (ii) variations in the capacity of information transfer mechanisms; and (iii) variations in the literacy, perceptual, cognitive and physical movement skills of the civilian.

\section{BACKGROUND}

\section{A. Emergency Warning Systems}

An Emergency Warning System (EWS) can play a crucial role in minimizing the impact of natural disasters by creating awareness amongst the population. Its primary use is to disseminate evacuation information to civilians, however, this can also extend to providing things like medical advice. The effectiveness of an EWS depends on several factors including the timeliness of the warning, its reachability (in terms of area and population) and its understandability.

In recent years, several countries have started deploying their own nationwide EWS. For instance, Japan has deployed the Earthquake and Tsunami Warning Service (ETWS) [5], whilst Indonesia has a similar service based on Twitter [3]. In Europe, the EU-Alert system [6] has also been proposed. A common feature of all of these systems is the use of commercially deployed networks, which are attractive due to their cost effective and widespread reach. However, these systems are typically built around one or two delivery systems, the most common ones being the short message service (SMS) and the cell broadcast service (CBS). While SMS is supported on every mobile phone, CBS support is not enabled by default in most phones. At the same time, both SMS and CBS place restrictions on the amount of information that can be carried in the payload. Recently, we have therefore seen the emergence of solutions built upon the public Internet, e.g. Google Alerts [7] and Twitter Alerts [8]. These leverage existing information services such as Google Maps and Twitter updates. Once again, these mechanisms focus narrowly on the subset of civilians who access the underlying service or application. While Google and Twitter have extensive reach in many parts of the world, the same cannot be said of many developing countries where Internet penetration is still relatively low. The bottom line is that no single mechanism is sufficient to cover all the possible deployment scenarios.

\section{B. Emergency Warning in Developing Countries}

Although our EWS is intended for deployment in many countries, in this paper we use India as a driving case study. Currently, coordinated support for disaster management in India is sadly lacking. Despite the setting up of a National Disaster Management Authority (NDMA) and its State-level counterpart (SDMA), there is still no effective EWS in place. Unfortunately, there is also a great degree of diversity amongst the population, e.g. literacy, linguistic and technological capabilities. These differences make a nationwide integrated EWS design highly challenging when compared to other countries (e.g. USA).

From a technological perspective, the primary challenge comes from the fact that the country still relies heavily upon GSM networks for cellular connectivity. Hence, some of the newer approaches like the 4G ETWS and the Commercial Mobile Alert System (used in the USA) are not feasible. Furthermore, the penetration of smartphones is still low, despite strong growth in recent years. Finally, Internet access is not widespread and limited to large and medium-sized towns and cities. Of course, a further problem remains which is that disaster situations are often accompanied by infrastructural collapse (e.g. damaged base stations). This is particularly prominent in India, where geographic spread makes infrastructure upkeep extremely difficult.

From a social perspective, there are two further challenges. First, India is a linguistically diverse country, with nearly two dozen officially recognized languages, spoken by millions, mostly on a regional basis. The second major issue is low literacy levels, which are only at $74 \%$ (65\% for females), rendering traditional text broadcast techniques useless. The bottom line is that the proposed EWS must take into account all of these technological and human-centric factors to ensure that an integrated solution can be deployed nationally.

\section{EMERGENCY WARNING SYSTEM}

In order to develop an EWS for operational use, it is important that the design process is not driven by innovation alone. Instead, it is now widely accepted that sufficient consideration should be given to social and human stakeholders. This section details an adaptive EWS (shown in Fig. 1), which we have designed following a human-centered approach [9]. The remainder of the paper is therefore based on outputs taken from a multinational stakeholder analysis, using interviews and observations with stakeholders including civilians, the NDMA, UK Mountain Rescue and the UK Fire Brigade. The rest of this section focusses on the technical components that operate on behalf of two of these stakeholders: authorities and civilians.

\section{A. Authorities}

\section{1) Warning Creation}

The authorities are a collection of organizations that manage the generation and distribution of evacuation warnings. These parties are linked by a hierarchy of control, shown in Fig. 1. At the highest level of the hierarchy is the EWS Coordinator. This is as a country-wide component and is situated within a virtualized cloud platform.

In essence, the EWS Coordinator is responsible for generating alerts. The first stage in this process is to monitor sensor data to help predict the likelihood of an emergency occurring. In India, the sensors are managed by various different agencies. For instance, the Central Water Commission is responsible for monitoring river levels while the Geological Survey of India generates data relating to seismic activity. Hence, based on the region and the type of disaster, these data sources will obviously vary. We are therefore neither 
prescriptive nor proscriptive in the form that these take but, instead, allow any form of sensor to be used. To improve interoperability, sensor information is presented in an XML form, alongside its XML schema. Errors with this can then be captured and dealt with by the EWS Administrator, who is responsible for managing the infrastructure.

On the receipt of a sensor reading, the EWS Coordinator computes whether a warning should be generated. Based on the disaster metadata (e.g. location and severity), several template warnings can be used, including pre-set information such as evacuations routes. However, this is not a fully automated process: an EWS Deployment Authority must manually authorize all warnings. Our current prototype adheres to the Common Alerting Protocol (CAP) standard, which defines an open XML format for writing evacuation warnings.

Once a warning has been authored, it is necessary to determine who should receive it (i.e. its distribution criteria). We allow the authorities to define distribution criteria over a variety of user attributes (e.g. demographics, location, cognitive abilities, and disabilities). This is formatted in an SQL-like SELECT query (e.g. user.age $<75$ ) to allow different user groups to receive different warnings. Using this, multiple targeted warnings can be sent to different groups of people.

\section{2) Warning Dissemination}

Following the stipulation of the distribution criteria, the warning is passed to one or more Dissemination Managers. These are independent services that are located and replicated across several geographically distinct data centers. They are responsible for ensuring that civilians under their domain of control receive the warning (typically, this domain is based on geography). Importantly, there is no assumption about any shared characteristics between these civilians. Thus, users could have access to different technologies and devices, have varying degrees of cognitive skills or even speak different languages. Further, certain parts of the network may have been damaged during the disaster meaning that not all dissemination mechanisms are operational. Due to the above constraint, sending a single warning over a single medium becomes unsuitable.

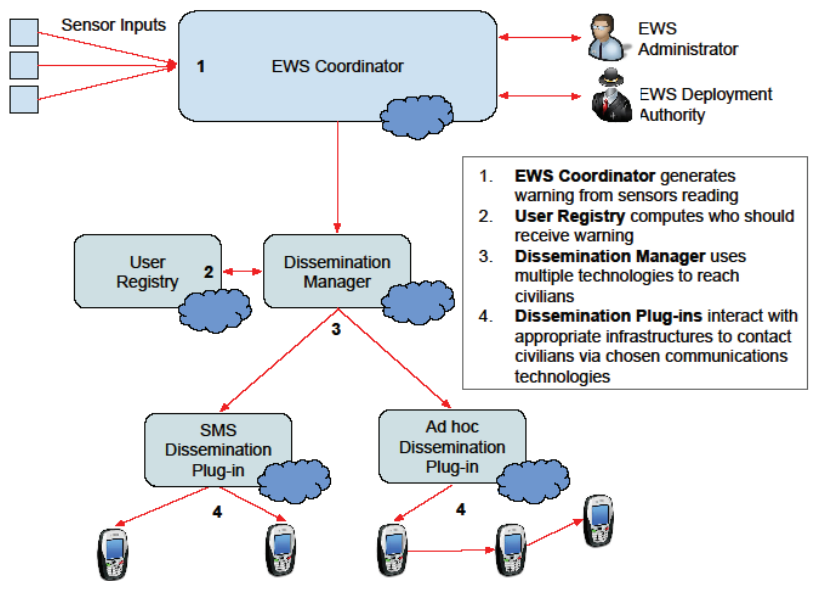

Fig. 1. An architectural overview of the EWS. Independent cloud services are highlighted using the cloud icon.
For example, an Internet-based delivery would not reach people with a basic features phone, nor would it reach people connected by damaged infrastructure. The Dissemination Managers try to address this by exploiting the observation that many devices have multiple interfaces, e.g. Bluetooth, Wi-Fi, $2 \mathrm{G} / 3 \mathrm{G}$. Using this, the Dissemination Managers dynamically re-configure between the uses of different dissemination technologies to reach users. As we later describe, this is achieved by maintaining a User Registry, to track individual users in the system.

To facilitate greater resilience, we view each Dissemination Manager as a replicated service that can exist in any of the data centers available to the EWS. This is shown in Fig. 2 with several distinct cloud data centers attached to the network. Despite the infrastructural damage at cloud A, the two users still retain connectivity with clouds B and C. Note that we are not proscriptive in how we define clouds; we also anticipate the deployment of small "cloudlets" that can operate near to the user, e.g. within mobile base stations.

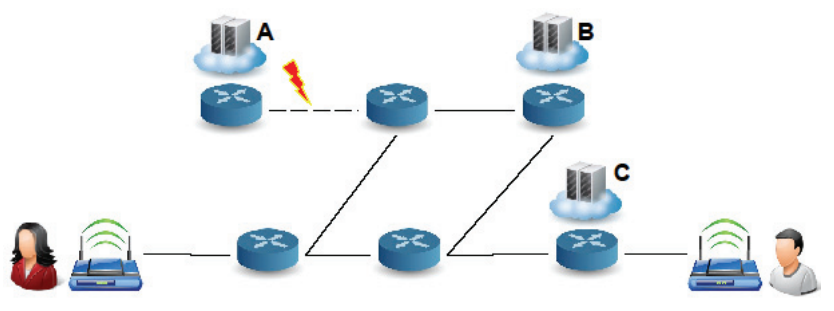

Fig. 2. Cloud-based infrastructure placement. The network connecting cloud $\mathrm{A}$ is damaged; the EWS continues to operate within clouds B and C.

\section{3) User Registration and Management}

To enable the above functionality, it is vital to retain a repository of civilian information. This is used by the Dissemination Managers to extract which civilians match the distribution criteria, as well as how they can be contacted (e.g. phone number). The User Registry contains a variety of information about registered users, including a user's language, visual acuity and motor skills, as well as a list of methods for communication (e.g. phone number, Twitter feeds they follow, email address). We later describe how this information is obtained. An entry is termed a user profile. Note that these profiles can be uploaded on behalf of individuals (e.g. automatically by their phone operator). To better facilitate this, not all information is mandatory: only geographical location and phone number. To improve resilience, a User Registry store is maintained alongside each Dissemination Manager, while a master registry is stored alongside the EWS Coordinator. Currently, all user profiles are replicated, however, to address scalability challenges this will likely need to change in the future.

To select which users should receive a given warning, the distribution criteria is applied to the user profile metadata stored within the registry to extract a set of users who the warning should be sent to. Once a set of recipients have been extracted, the Dissemination Managers then compute the optimal dissemination scheme to contact them through (described later). 


\section{B. Civilians}

A civilian is anybody who wishes to receive warnings. To assist them, we have developed an EWS Android app. However, to fulfil our goal of high reachability, we make no assumption regarding a civilian's possession of the app (for example, in India there is only an $18 \%$ penetration of smartphones). We therefore group civilians into two brackets: (i) app-enabled: users who have downloaded the app onto their smartphone; and (ii) legacy: users who do not have access to the app.

To differentiate between app-enabled and legacy users, the User Registry retains information about the devices carried by individuals. Those without the app will always receive plain text warnings for immediate viewing via in-built communication mechanisms (e.g. SMS), whereas users with the app will receive warnings in a computable format that can be rendered to the screen in a more sophisticated manner. The smartphone app, in conjunction with the Dissemination Managers currently supports the following dissemination mechanisms:

- SMS: The app can receive warnings via SMS. This is limited to basic text of 160 characters and is therefore only used when data connectivity is not available.

- AMQP: The app can receive warnings via Advanced Message Queuing Protocol (AMQP), which is a publish/subscribe protocol, running over IP. This offers the transmission of large bodies of text, audio, image and video, which is the preferred means for all smartphone users.

- Ad hoc: The app can form an ad hoc Wi-Fi network between phones when all infrastructure has collapsed. This allows responders to move through areas disseminating the warning in a peer-to-peer fashion. It is only used when SMS and AMQP schemes are unavailable, as it is inherently opportunistic.

Importantly, based on the device's Dissemination Manager, the app will seamlessly switch between the uses of these technologies to best optimize the warning delivery. In all of the above cases, app-enabled phones can render the warnings in an easy-to-read manner, as shown in Fig. 3.

\section{EWS ADAPTATION}

The previous section has given a high-level overview of an adaptive EWS. We now focus on three types of adaptation employed.

\section{A. Dissemination Adaptation}

During disaster situations, it is commonplace for infrastructural failures to occur [4]. This might, for example, result in parts of the GSM network collapsing.

To address this, the Dissemination Manager instances dynamically re-configures between the use of different dissemination schemes on-demand (e.g. SMS, AMQP, Twitter).

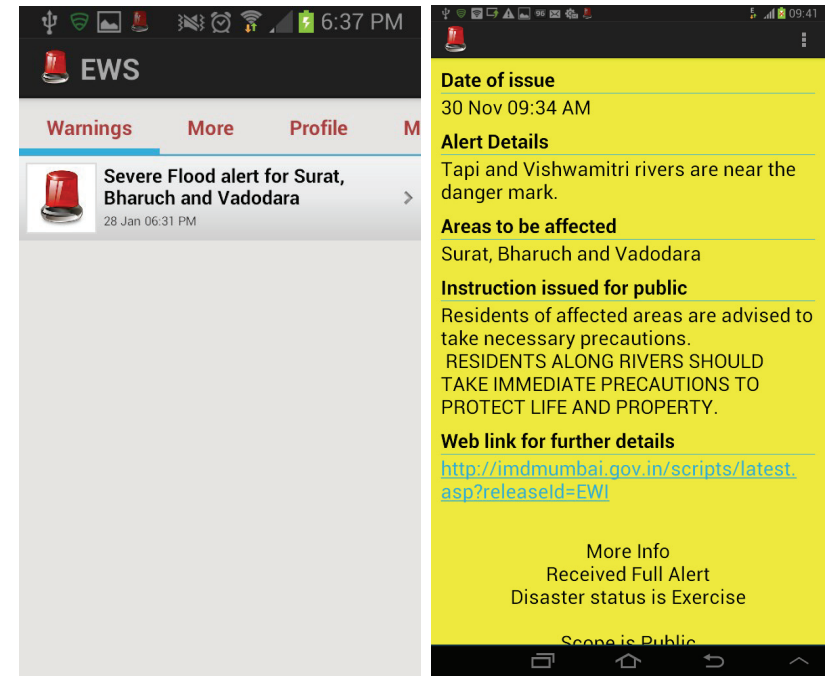

Fig. 3. The EWS Android App. On the left is a list of all warnings received, with normal colour rendering. On the right is a description of a particular warning, with yellow rendering for Dichromatic colour blindness.

This flexibility allows the Dissemination Managers to avoid such failures, exploiting the multi-interface access offered by many civilians and their devices. Further, the ability to dynamically place instances of the Dissemination Manager in any virtualized network location available means that they can be situated in topological positions that still possess connectivity with the maximum number of civilians.

Each dissemination scheme is encapsulated within a cloud plug-in service that offers the functionality to transport a variable sized block of information from an origin (the authorities) to a destination (a civilian). Current plug-ins are for SMS, AMQP and ad hoc. These are used by the instances of the Dissemination Manager to contact civilians. A Dissemination Manager selects which plug-in to use via a Policy Repository. Policies consist of simple rule sets that are executed over metadata that describes the disaster, the civilian(s) to be contacted and the available infrastructure. The execution of the policy rule set returns a selected dissemination strategy, which informs the Dissemination Manager how to contact each civilian and in what order. An example is given in Fig. 4. The relevant metadata is easy to obtain: (i) Disaster metadata is retrieved from the actual CAP warning itself, as this lists things like urgency, certainty and scope; (ii) Civilian metadata is obtained from the User Registry; and (iii) Infrastructure metadata is obtained from the plug-ins, which each provide information on their operational conditions (e.g. status, bandwidth capacity, MIME types supported).

These three sets of metadata are utilized by the Dissemination Managers to decide how to contact each civilian. Dissemination strategies are structured as scripts listing the order of plug-ins to try for each civilian. For example, policies can be defined so that in high risk settings, any broadcast-enabled plug-ins will be used. 


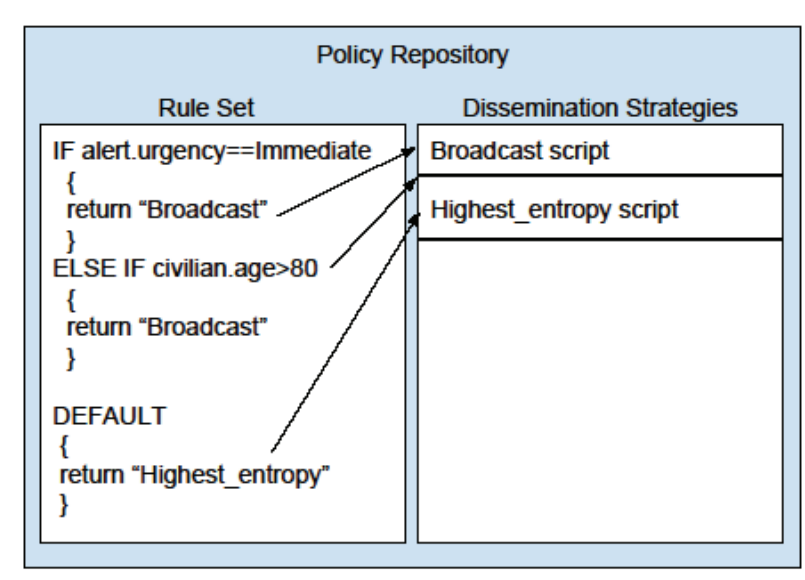

Fig. 4. A simple example of the Policy Repository.

Currently, two dissemination strategies have been implemented: (i) broadcast and (ii) highest entropy. Broadcast is the simplest option, in which each civilian is contacted via all mechanisms available to them. Highest entropy, instead, selects (for each civilian) the single dissemination mechanism with the highest level of entropy (for example, AMQP dissemination can transport more information than an SMS). If the mechanism fails, the next best dissemination technique is tried. This runtime approach avoids the need to perform constant monitoring of each civilian, instead allowing mechanisms to be experimented with until a success is achieved. Note that this adaptation not only addresses communications failures on the civilian's device, but also intermediate failures in the rest of the network.

\section{B. Information Adaptation}

The above form of delivery adaptation creates a significant challenge. Whereas some delivery schemes (e.g. AMQP) are extremely flexible with the ability to send text, images and videos, others are far more limited in their capabilities (e.g. SMS). On the one hand, all warnings could address the lowest common denominator by only sending short text. Whereas, on the other hand, they could include multimedia but limit the warning's applicability to civilians with Internet-enabled smartphones. Both cases are unacceptable in situations where device and user diversity is high (e.g. India).

To address this, warnings are adapted for different users, allowing individuals to best exploit their ability to receive and render complex content messages. Each type of warning is augmented with a Serializer that must be defined in advance. This is responsible for serializing warnings for transmission. As parameters, it accepts the dissemination mechanism and recipient(s) it is serializing for. It must then serialize the warning in a way that is most appropriate. In our current prototype, the Serializer for our CAP warnings captures all elements when disseminating using AMQP or ad hoc. For SMS, however, it condenses warnings to only include the most important textual parts: the sender and evacuation description. Importantly, due to India's language diversity, the Serializer also performs translation to the recipient's preferred language. Thus, warnings are, by default, provided in English and then transformed into an appropriate language for the civilian a priori.

\section{Presentation Adaptation}

Our human-centered design strategy highlighted a huge diversity in user capabilities. For example, in India and the UK many people in rural and urban populations have varying literacy, visual, cognitive and motor capabilities. We argue that an EWS must be inclusive, addressing these issues, particularly as those with age related or physical impairment are more vulnerable.

To handle the above concerns, a user modelling application has been developed (shown in Fig. 5) to adapt civilians' EWS user interfaces. It is offered as part of the registration procedure and asks users to perform a small set of tasks that help the system model physical capability and visual acuity. This generates a user capability profile consistent with EU and ITU standards [10]. The profile is then processed by the smartphone app to dynamically render warnings, adapting such things as font size, color contrast, zooming level, button layout and line spacing.

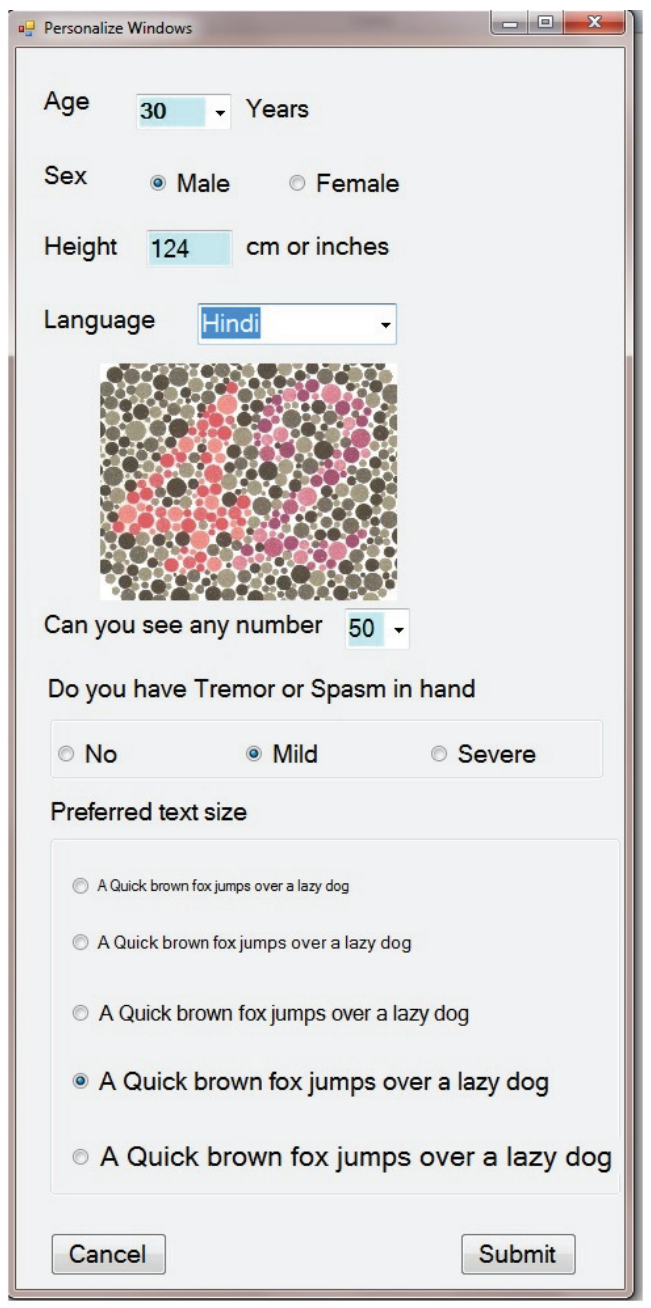

Fig. 5. The Personalisation interface for generating the user model. 
For example, it changes background and foreground colors for color blind users, turns on a text-to-speech converter for visually impaired users and increases line spacing and default zooming level for users with a tremor or spasm in the hand. An example of this can be seen in Fig. 3, where different color coding is used to address Dichromatic color blindness. Through this, we can ensure that a variety of users can interact with the EWS. The user modelling system has been extensively validated with user trials.

\section{CONCLUSION}

This paper has detailed a cloud-based EWS targeted at heterogeneous technological and sociological environments, e.g. India. This has taken place as part of a collaboration between the UK and India. Our prototype is ready for trial in India and the UK, but parts of the system have already been trialed. The presentation adaptation has been trialed in India with interesting results [12]. In the UK, QMUL and Cambridge performed a trial in the Lake District where the EWS manager was tested making use of ad hoc communication. The scenario used for the trial depicted an emergency (e.g. heavy snow) where civilians were trapped in their homes in a radio dead zone village and a rescue team would pass by their houses; in the tested trial, the EWS app in the civilian phone was able to send a SOS and the rescue team's EWS app was able to send a message/warning to the civilian. The test worked successfully for a rescue personnel walking closely to the house, but failed if passing by car. Trials with the full system is then likely to yield a number of interesting avenues of future work. However, we already foresee a number of extensions that could be investigated. First, more sophisticated dissemination plug-ins, in conjunction with dissemination strategies, should be developed. We are particularly interested in integrating future technologies into this process, including things like information-centric networks [13] and more advanced delay tolerant networks [14]. It is also necessary to develop more sophisticated placement strategies to ensure that connectivity between cloud instances and civilians remains high.

\section{REFERENCES}

[1] "Natural disasters up more than 400 percent in two decades." http://www.naturalnews.com/ 023362 natural disasters floods.html.

[2] S. Ganeshan and W. Diamond, "Forecasting the numbers of people affected annually by natural disasters up to 2015," Oxfam Report, 2009.

[3] A. T. Chatfield and U. Brajawidagda, "Twitter early tsunami warning system: A case study in indonesia's natural disaster management," in System Sciences (HICSS), 2013 46th Hawaii International Conference on, pp. 2050-2060, 2013.

[4] K. Cho, C. Pelsser, R. Bush, and Y. Won, "The Japan earthquake: the impact on traffic and routing observed by a local ISP," in Proceedings of the Special Workshop on Internet and Disasters, p. 2, ACM, 2011.

[5] "Earthquake and tsunami warning service." http://www.jma.go.jp/en/tsunami/.

[6] "European public warning system using the cell broadcast service (eualert).” ETSI Technical Specification ES102.900.

[7] "Google public alerts." https://www.google.org/crisisresponse/publicalerts/.

[8] G. Pena, "Twitter disaster alerts." Twitter Alerts: Critical information when you need it most, 2013.

[9] P. Langdon, "Developing an interactive tv for the elderly and impaired: An inclusive design strategy," in A Multimodal End-2-End Approach to Accessible Computing, pp. 23-48, Springer, 2013.

[10] ITU, "Technical report of the focus group on smart cable television." http://www.itu.int.

[11] P. Biswas, P. Robinson, and P. Langdon, "Designing inclusive interfaces through user modeling and simulation," International Journal of HumanComputer Interaction, vol. 28, no. 1, pp. 1-33, 2012.

[12] P. Biswas, P. Langdon, S. Sarangi, V. Mimrot, S. Kar, V. J. Ribeiro, J. Umadikar, S. Prashant, "Interface Personalization through Inclusive User Modelling Web Service," Proc. of the XV International Conference on Human Computer Interaction, article number 103, September 10-12, 2014. Puerto de la Cruz, Tenerife, Spain.

[13] K. Pentikousis, B. Ohlman, D. Corujo, G. Boggia,G. Tyson, E. Davies, D. Gellert, and P. Mahadevan, "Information-centric networking: Baseline scenarios," Internet Draft, 2013.

[14] G. Tyson, J. Bigham, and E. Bodanese, "Towards an information-centric delay-tolerant network," in Proc. IEEE INFOCOM Workshop on Emerging Design Choices in Name-Oriented Networking (NOMEN), 2013. 